\title{
Some Inequalities of Simpson Type for $h$-Convex Functions via Fractional Integrals
}

\author{
Marian Matłoka \\ Department of Applied Mathematics, Poznań University of Economics, Aleja Niepodległości 10, 61-875 Poznań, Poland \\ Correspondence should be addressed to Marian Matłoka; marian.matloka@ue.poznan.pl
}

Received 21 March 2015; Accepted 1 July 2015

Academic Editor: Alberto Fiorenza

Copyright (C) 2015 Marian Matłoka. This is an open access article distributed under the Creative Commons Attribution License, which permits unrestricted use, distribution, and reproduction in any medium, provided the original work is properly cited.

We establish some inequalities of Simpson type involving Riemann-Liouville fractional integrals for mappings whose first derivatives are $h$-convex.

\section{Introduction}

The following inequality is well known in the literature as Simpson's inequality.

Theorem 1. Let $f:[a, b] \rightarrow R$ be four times continuously differentiable mapping on $(a, b)$ and $\left\|f^{(4)}\right\|_{\infty}=$ $\sup _{x \in(a, b)}\left|f^{(4)}(x)\right|<\infty$.

Then, the following inequality holds:

$$
\begin{aligned}
& \left|\frac{1}{3}\left[\frac{f(a)+f(b)}{2}+2 f\left(\frac{a+b}{2}\right)\right]-\frac{1}{b-a} \int_{a}^{b} f(x) d x\right| \\
& \quad \leq \frac{1}{2880}\left\|f^{(4)}\right\|_{\infty}(b-a)^{4} .
\end{aligned}
$$

In [1], Dragomir et al. proved the following inequality.

Theorem 2. Suppose $f:[a, b] \rightarrow R$ is a differentiable mapping whose derivative is continuous on $(a, b)$ and $f^{\prime} \in$ $L_{1}[a, b]$. Then the following inequality holds:

$$
\begin{aligned}
& \mid \frac{1}{3}\left[\frac{f(a)+f(b)}{2}+2 f\left(\frac{a+b}{2}\right)\right] \\
& -\frac{1}{b-a} \int_{a}^{b} f(x) d x \mid \leq \frac{b-a}{3}\left\|f^{\prime}\right\|_{1},
\end{aligned}
$$

where $\left\|f^{\prime}\right\|_{1}=\int_{a}^{b}\left|f^{\prime}(x)\right| d x$.
In [2], Sarikaya et al. obtained inequalities for differentiable convex mappings. The main inequality is as follows.

Theorem 3. Let $f: I \subset R \rightarrow R$ be a differentiable mapping on $I^{\circ}$ such that $f^{\prime} \in L_{1}[a, b]$, where $a, b \in I^{\circ}$ with $a<b$. If $\left|f^{\prime}\right|^{q}$ is convex on $[a, b], q>1$, then the following inequality holds:

$$
\begin{aligned}
& \left|\frac{1}{6}\left[f(a)+4 f\left(\frac{a+b}{2}\right)+f(b)\right]-\frac{1}{b-a} \int_{a}^{b} f(x) d x\right| \\
& \quad \leq \frac{b-a}{12}\left(\frac{1+2^{p+1}}{3(p+1)}\right)^{1 / p} \\
& \quad+\left\{\frac{3\left|f^{\prime}(b)\right|^{q}+\left|f^{\prime}(a)\right|^{q}}{4}\right)^{1 / q} \\
& \left.+\left(\frac{\left|f^{\prime}(b)\right|^{q}+3\left|f^{\prime}(a)\right|^{q}}{4}\right)^{1 / q}\right\}
\end{aligned}
$$

where $1 / p+1 / q=1$.

In [3], Sarikaya et al. obtained the following inequality for $s$-convex function. 
Theorem 4. Let $f: I \subset[0, \infty] \rightarrow R$ be a differentiable mapping on $I^{\circ}$ such that $f^{\prime} \in L_{1}[a, b]$, where $a, b \in I^{\circ}$ with $a<b$. If $\left|f^{\prime}\right|^{q}$ is s-convex on $[a, b]$, for some fixed $s \in[0,1]$ and $q>1$, then the following inequality holds:

$$
\begin{aligned}
& \left|\frac{1}{6}\left[f(a)+4 f\left(\frac{a+b}{2}\right)+f(b)\right]-\frac{1}{b-a} \int_{a}^{b} f(x) d x\right| \\
& \leq \frac{(b-a)}{12}\left(\frac{1+2^{p+1}}{3(p+1)}\right)^{1 / p} \\
& \cdot\left\{\left(\frac{\left|f^{\prime}(b)\right|^{q}+\left|f^{\prime}((a+b) / 2)\right|^{q}}{s+1}\right)^{1 / q}\right. \\
& \left.+\left(\frac{\left|f^{\prime}(a)\right|^{q}+\left|f^{\prime}((a+b) / 2)\right|^{q}}{s+1}\right)^{1 / q}\right\}
\end{aligned}
$$

where $1 / p+1 / q=1$

For recent refinements, counterparts, generalizations, and inequalities of Simpson type, see [1-7].

In 2007, Varošanec in [8] introduced a large class of functions, the so-called $h$-convex functions. This class contains several well-known classes of functions such as nonnegative convex functions and $s$-convex functions. This class is defined in the following way: a function $f: I \rightarrow R, \emptyset \neq I \subset R$ being an interval, is called $h$-convex if

$$
f(t x+(1-t) y) \leq h(t) f(x)+h(1-t) f(y)
$$

holds for all $x, y \in I, t \in(0,1)$, where $h: J \rightarrow R, h \neq \equiv$, and $J$ is an interval, $(0,1) \subseteq J$.

The aim of this paper is to establish inequalities of Simpson type for $h$-convex mappings via fractional integrals which are defined in the following way: left-sided and rightsided Riemann-Liouville fractional integrals of the order $\alpha>$ 0 are defined by

$$
\begin{aligned}
& I_{a^{+}}^{\alpha} f(x)=\frac{1}{\Gamma(\alpha)} \int_{a}^{x}(x-t)^{\alpha-1} f(t) d t \\
& \quad(0 \leq a<x \leq b), \\
& I_{b^{-}}^{\alpha} f(x)=\frac{1}{\Gamma(\alpha)} \int_{x}^{b}(t-x)^{\alpha-1} f(t) d t \\
& \quad(0 \leq a \leq x<b),
\end{aligned}
$$

where $\Gamma(\alpha)=\int_{0}^{\infty} e^{-u} u^{\alpha-1} d u$ is the gamma function. Here is $I_{a^{+}}^{\circ} f(x)=I_{b^{-}}^{\circ} f(x)=f(x)$.

\section{Main Results}

To prove our main results, we consider the following lemma.

Lemma 5. Let $f: I \subset R \rightarrow R$ be an absolutely continuous mapping on $I^{\circ}$ such that $f^{\prime} \in L_{1}[a, b]$, where $a, b \in I^{\circ}$ with $a<b$. Then the following inequality holds:

$$
\begin{aligned}
& \frac{1}{6}\left[f(a)+4 f\left(\frac{a+b}{2}\right)+f(b)\right] \\
& -\frac{2^{\alpha-1} \Gamma(\alpha+1)}{(b-a)^{\alpha}}\left[I_{a^{+}}^{\alpha} f\left(\frac{a+b}{2}\right)+I_{b^{-}}^{\alpha} f\left(\frac{a+b}{2}\right)\right] \\
& \quad=\frac{b-a}{2}\left[\int_{0}^{1}\left(\frac{t^{\alpha}}{2}-\frac{1}{3}\right) f^{\prime}\left(\frac{1+t}{2} b+\frac{1-t}{2} a\right) d t\right. \\
& \left.\quad+\int_{0}^{1}\left(\frac{1}{3}-\frac{t^{\alpha}}{2}\right) f^{\prime}\left(\frac{1+t}{2} a+\frac{1-t}{2} b\right) d t\right] .
\end{aligned}
$$

Proof. By integration by parts and by the change of the variables, we have

$$
\begin{aligned}
& \int_{0}^{1}\left(\frac{t^{\alpha}}{2}-\frac{1}{3}\right) f^{\prime}\left(\frac{1+t}{2} b+\frac{1-t}{2} a\right) d t \\
&=\left.\frac{2}{b-a}\left(\frac{t^{\alpha}}{2}-\frac{1}{3}\right) f\left(\frac{1+t}{2} b+\frac{1-t}{2} a\right)\right|_{0} ^{1} \\
&-\frac{\alpha}{b-a} \int_{0}^{1} t^{\alpha-1} f\left(\frac{1+t}{2} b+\frac{1-t}{2} a\right) d t \\
&= \frac{1}{3(b-a)} f(b)+\frac{2}{3(b-a)} f\left(\frac{a+b}{2}\right) \\
&-\frac{2^{\alpha} \cdot \alpha}{(b-a)^{\alpha+1}} \int_{(a+b) / 2}^{b}\left(u-\frac{a+b}{2}\right)^{\alpha-1} f(u) d u \\
&= \frac{1}{3(b-a)} f(b)+\frac{2}{3(b-a)} f\left(\frac{a+b}{2}\right) \\
&-\frac{2^{\alpha} \Gamma(\alpha+1)}{(b-a)^{\alpha+1}} I_{b^{-}}^{\alpha} f\left(\frac{a+b}{2}\right) .
\end{aligned}
$$

Similarly, we have

$$
\begin{aligned}
\int_{0}^{1}( & \left.\frac{1}{3}-\frac{t^{\alpha}}{2}\right) f^{\prime}\left(\frac{1+t}{2} a+\frac{1-t}{2} b\right) d t \\
= & \left.\frac{2}{a-b}\left(\frac{1}{3}-\frac{t^{\alpha}}{2}\right) f\left(\frac{1+t}{2} a+\frac{1-t}{2} b\right)\right|_{0} ^{1} \\
& +\frac{\alpha}{a-b} \int_{0}^{1} t^{\alpha-1} f\left(\frac{1+t}{2} a+\frac{1-t}{2} b\right) d t \\
= & \frac{1}{3(b-a)} f(a)+\frac{2}{3(b-a)} f\left(\frac{a+b}{2}\right) \\
& +\frac{2^{\alpha} \cdot \alpha}{(b-a)^{\alpha+1}} \int_{(a+b) / 2}^{a}\left(\frac{a+b}{2}-u\right)^{\alpha-1} f(u) d u \\
= & \frac{1}{3(b-a)} f(a)+\frac{2}{3(b-a)} f\left(\frac{a+b}{2}\right) \\
& -\frac{2^{\alpha} \Gamma(\alpha+1)}{(b-a)^{\alpha+1}} I_{a^{+}}^{\alpha} f\left(\frac{a+b}{2}\right) .
\end{aligned}
$$

From (8) and (9), we get (7). This completes the proof. 
The following theorems give a new result of Simpson's inequality for $h$-convex functions via fractional integrals.

Theorem 6. Let $f: I \subset R \rightarrow R$ be a differentiable mapping on $I^{\circ}$ such that $f^{\prime} \in L_{1}[a, b]$, where $a, b \in I^{\circ}$ with $a<b$. If $\left|f^{\prime}\right|$ is h-convex on $[a, b]$, then the following inequality holds:

$$
\begin{aligned}
& \mid \frac{1}{6}\left[f(a)+4 f\left(\frac{a+b}{2}\right)+f(b)\right] \\
& -\frac{2^{\alpha-1} \Gamma(\alpha+1)}{(b-a)^{\alpha}}\left[I_{a^{+}}^{\alpha} f\left(\frac{a+b}{2}\right)+I_{b^{-}}^{\alpha} f\left(\frac{a+b}{2}\right)\right] \mid \\
& \quad \leq \frac{b-a}{3}\left[\left|f^{\prime}(a)\right|+\left|f^{\prime}(b)\right|\right] \int_{0}^{1} h(t) d t .
\end{aligned}
$$

Proof. From Lemma 5 and since $\left|f^{\prime}\right|$ is $h$-convex on $[a, b]$, we get

$$
\begin{aligned}
& \mid \frac{1}{6}\left[f(a)+4 f\left(\frac{a+b}{2}\right)+f(b)\right] \\
& \quad-\frac{2^{\alpha-1} \Gamma(\alpha+1)}{(b-a)^{\alpha}}\left[I_{a^{+}}^{\alpha} f\left(\frac{a+b}{2}\right)+I_{b^{-}}^{\alpha} f\left(\frac{a+b}{2}\right)\right] \mid \\
& \quad \leq \frac{b-a}{2} \int_{0}^{1}\left[\left|\frac{t^{\alpha}}{2}-\frac{1}{3}\right|\left|f^{\prime}\left(\frac{1+t}{2} b+\frac{1-t}{2} a\right)\right|\right. \\
& \left.\quad+\left|\frac{1}{3}-\frac{t^{\alpha}}{2}\right|\left|f^{\prime}\left(\frac{1+t}{2} a+\frac{1-t}{2} b\right)\right|\right] d t \leq \frac{b-a}{2} \\
& \quad \cdot \int_{0}^{1}\left|\frac{t^{\alpha}}{2}-\frac{1}{3}\right|\left[h\left(\frac{1+t}{2}\right)\left|f^{\prime}(b)\right|\right. \\
& \quad+h\left(\frac{1-t}{2}\right)\left|f^{\prime}(a)\right|+h\left(\frac{1+t}{2}\right)\left|f^{\prime}(a)\right| \\
& \left.\quad+h\left(\frac{1-t}{2}\right)\left|f^{\prime}(b)\right|\right] d t=\frac{b-a}{2}\left[\left|f^{\prime}(a)\right|\right. \\
& \left.\quad+\left|f^{\prime}(b)\right|\right] \int_{0}^{1}\left|\frac{t^{\alpha}}{2}-\frac{1}{3}\right|\left[h\left(\frac{1+t}{2}\right)+h\left(\frac{1-t}{2}\right)\right] d t \\
& \quad \leq \frac{b-a}{3}\left[\left|f^{\prime}(a)\right|+\left|f^{\prime}(b)\right|\right] \int_{0}^{1} h(t) d t,
\end{aligned}
$$

where we used the fact that $\left|t^{\alpha} / 2-1 / 3\right| \leq 1 / 3$ for all $x \in[0,1]$. The proof is completed.

Corollary 7. If in Theorem 6 one takes $h(t)=t$ then inequality (10) reduces to the following inequality for the convex function:

$$
\begin{aligned}
& \mid \frac{1}{6}\left[f(a)+4 f\left(\frac{a+b}{2}\right)+f(b)\right] \\
& -\frac{2^{\alpha-1} \Gamma(\alpha+1)}{(b-a)^{\alpha}}\left[I_{a^{+}}^{\alpha} f\left(\frac{a+b}{2}\right)+I_{b^{-}}^{\alpha} f\left(\frac{a+b}{2}\right)\right] \mid \\
& \quad \leq \frac{b-a}{6}\left[\left|f^{\prime}(a)\right|+\left|f^{\prime}(b)\right|\right] .
\end{aligned}
$$

Corollary 8. If in Theorem 6 one takes $h(t)=t^{s}$ then inequality (10) reduces to the following inequality for the $s$ convex function:

$$
\begin{aligned}
& \mid \frac{1}{6}\left[f(a)+4 f\left(\frac{a+b}{2}\right)+f(b)\right] \\
& -\frac{2^{\alpha-1} \Gamma(\alpha+1)}{(b-a)^{\alpha}}\left[I_{a^{+}}^{\alpha} f\left(\frac{a+b}{2}\right)+I_{b^{-}}^{\alpha} f\left(\frac{a+b}{2}\right)\right] \mid \\
& \quad \leq \frac{b-a}{3(s+1)}\left[\left|f^{\prime}(a)\right|+\left|f^{\prime}(b)\right|\right] .
\end{aligned}
$$

Corollary 9. If in Theorem 6 one takes $h(t)=t$ and $\alpha=1$ then from the proof of Theorem 6 it follows that the following inequality holds:

$$
\begin{aligned}
& \mid \frac{1}{6}\left[f(a)+4 f\left(\frac{a+b}{2}\right)+f(b)\right] \\
& -\frac{1}{b-a} \int_{a}^{b} f(x) d x \mid \leq \frac{5(b-a)}{72}\left[\left|f^{\prime}(a)\right|\right. \\
& \left.+\left|f^{\prime}(b)\right|\right]
\end{aligned}
$$

Theorem 10. Let $f: I \subset R \rightarrow R$ be a differentiable mapping on $I^{\circ}$ such that $f^{\prime} \in L_{1}[a, b]$, where $a, b \in I^{\circ}$ with $a<b$. If $\left|f^{\prime}\right|^{q}$ is $h$-convex on $[a, b]$ and $q>1$, then the following inequality holds:

$$
\begin{aligned}
& \mid \frac{1}{6}\left[f(a)+4 f\left(\frac{a+b}{2}\right)+f(b)\right] \\
& \quad-\frac{2^{\alpha-1} \Gamma(\alpha+1)}{(b-a)^{\alpha}}\left[I_{a^{+}}^{\alpha} f\left(\frac{a+b}{2}\right)+I_{b^{-}}^{\alpha} f\left(\frac{a+b}{2}\right)\right] \mid \\
& \quad \leq \frac{b-a}{6}\left\{\left[\left|f^{\prime}(b)\right|^{q} \int_{0}^{1} h\left(\frac{1+t}{2}\right) d t\right.\right. \\
& \left.\quad+\left|f^{\prime}(a)\right|^{q} \int_{0}^{1} h\left(\frac{1-t}{2}\right) d t\right]^{1 / q} \\
& \quad+\left[\left|f^{\prime}(a)\right|^{q} \int_{0}^{1} h\left(\frac{1+t}{2}\right) d t\right. \\
& \left.\left.\quad+\left|f^{\prime}(b)\right|^{q} \int_{0}^{1} h\left(\frac{1-t}{2}\right) d t\right]^{1 / q}\right\} .
\end{aligned}
$$


Proof. From Lemma 5 and the Hölder inequality, we have

$$
\begin{aligned}
& \mid \frac{1}{6}\left[f(a)+4 f\left(\frac{a+b}{2}\right)+f(b)\right] \\
& \quad-\frac{2^{\alpha-1} \Gamma(\alpha+1)}{(b-a)^{\alpha}}\left[I_{a^{+}}^{\alpha} f\left(\frac{a+b}{2}\right)+I_{b^{-}}^{\alpha} f\left(\frac{a+b}{2}\right)\right] \mid \\
& \quad \leq \frac{b-a}{2}\left\{\left(\int_{0}^{1}\left|\frac{t^{\alpha}}{2}-\frac{1}{3}\right|^{p} d t\right)^{1 / p}\right. \\
& \quad \cdot\left(\int_{0}^{1}\left|f^{\prime}\left(\frac{1+t}{2} b+\frac{1-t}{2} a\right)\right|^{q} d t\right)^{1 / q} \\
& \quad+\left(\int_{0}^{1}\left|\frac{1}{3}-\frac{t^{\alpha}}{2}\right|^{p} d t\right)^{1 / p} \\
& \left.\quad \cdot\left(\int_{0}^{1}\left|f^{\prime}\left(\frac{1+t}{2} a+\frac{1-t}{2} b\right)\right|^{q} d t\right)^{1 / q}\right\}
\end{aligned}
$$

where $1 / p+1 / q=1$.

Because $\left|f^{\prime}\right|^{q}$ is $h$-convex, we have

$$
\begin{gathered}
\int_{0}^{1}\left|f^{\prime}\left(\frac{1+t}{2} b+\frac{1-t}{2} a\right)\right|^{q} d t \\
\leq\left|f^{\prime}(b)\right|^{q} \int_{0}^{1} h\left(\frac{1+t}{2}\right) d t \\
+\left|f^{\prime}(a)\right|^{q} \int_{0}^{1} h\left(\frac{1-t}{2}\right) d t \\
\int_{0}^{1}\left|f^{\prime}\left(\frac{1+t}{2} a+\frac{1-t}{2} b\right)\right|^{q} d t \\
\leq\left|f^{\prime}(a)\right|^{q} \int_{0}^{1} h\left(\frac{1+t}{2}\right) d t \\
+\left|f^{\prime}(b)\right|^{q} \int_{0}^{1} h\left(\frac{1-t}{2}\right) d t .
\end{gathered}
$$

Using the fact that $\left|t^{\alpha} / 2-1 / 3\right|=\left|1 / 3-t^{\alpha} / 2\right| \leq 1 / 3$ for all $t \in[0,1]$ and using the last two inequalities in (16) we obtain (15).

This completes the proof of the theorem.

Corollary 11. If in Theorem 10 one takes $h(t)=t$ then inequality (15) reduces to the following inequality for the convex function:

$$
\begin{aligned}
& \mid \frac{1}{6}\left[f(a)+4 f\left(\frac{a+b}{2}\right)+f(b)\right] \\
& \quad-\frac{2^{\alpha-1} \Gamma(\alpha+1)}{(b-a)^{\alpha}}\left[I_{a^{+}}^{\alpha} f\left(\frac{a+b}{2}\right)+I_{b^{-}}^{\alpha} f\left(\frac{a+b}{2}\right)\right] \mid \\
& \quad \leq \frac{b-a}{2}\left[\left(\frac{3}{4}\left|f^{\prime}(b)\right|^{q}+\frac{1}{4}\left|f^{\prime}(a)\right|^{q}\right)^{1 / q}\right. \\
& \left.\quad+\left(\frac{1}{4}\left|f^{\prime}(b)\right|^{q}+\frac{3}{4}\left|f^{\prime}(a)\right|^{q}\right)^{1 / q}\right] .
\end{aligned}
$$

Corollary 12. If in Theorem 10 one takes $h(t)=t^{s}$ then inequality (15) reduces to the following inequality for the sconvex function:

$$
\begin{aligned}
& \mid \frac{1}{6}\left[f(a)+4 f\left(\frac{a+b}{2}\right)+f(b)\right] \\
& \quad-\frac{2^{\alpha-1} \Gamma(\alpha+1)}{(b-a)^{\alpha}}\left[I_{a^{+}}^{\alpha} f\left(\frac{a+b}{2}\right)+I_{b^{-}}^{\alpha} f\left(\frac{a+b}{2}\right)\right] \mid \\
& \quad \leq \frac{b-a}{2}\left(\frac{2}{s+1}\right)^{1 / q} \\
& \quad .\left\{\left(\left|f^{\prime}(b)\right|^{q}\left(1-\frac{1}{2^{s+1}}\right)+\left|f^{\prime}(a)\right|^{q} \frac{1}{2^{s+1}}\right)^{1 / q}\right. \\
& \left.\quad+\left(\left|f^{\prime}(b)\right|^{q} \frac{1}{2^{s+1}}+\left|f^{\prime}(a)\right|^{q}\left(1-\frac{1}{2^{s+1}}\right)\right)^{1 / q}\right\} .
\end{aligned}
$$

Theorem 13. Let $f: I \subset R \rightarrow R$ be a differentiable mapping on $I^{\circ}$ such that $f^{\prime} \in L_{1}[a, b]$, where $a, b \in I^{\circ}$ with $a<b$. If $\left|f^{\prime}\right|^{q}$ is $h$-convex on $[a, b]$ and $q \geq 1$, then the following inequality holds:

$$
\begin{aligned}
& \mid \frac{1}{6}\left[f(a)+4 f\left(\frac{a+b}{2}\right)+f(b)\right] \\
& \quad-\frac{2^{\alpha-1} \Gamma(\alpha+1)}{(b-a)^{\alpha}}\left[I_{a^{+}}^{\alpha} f\left(\frac{a+b}{2}\right)+I_{b^{-}}^{\alpha} f\left(\frac{a+b}{2}\right)\right] \mid \\
& \quad \leq \frac{b-a}{2}\left\{\left(\left|f^{\prime}(b)\right|^{q} \int_{0}^{1} h\left(\frac{1+t}{2}\right) d t\right.\right. \\
& \left.\quad+\left|f^{\prime}(a)\right|^{q} \int_{0}^{1} h\left(\frac{1-t}{2}\right) d t\right)^{1 / q} \\
& \quad+\left(\left|f^{\prime}(a)\right|^{q} \int_{0}^{1} h\left(\frac{1+t}{2}\right) d t\right. \\
& \left.\left.\quad+\left|f^{\prime}(b)\right|^{q} \int_{0}^{1} h\left(\frac{1-t}{2}\right) d t\right)^{1 / q}\right\} .
\end{aligned}
$$

Proof. From Lemma 5 and the power mean inequality, we have that the following inequality holds:

$$
\begin{aligned}
& \mid \frac{1}{6}\left[f(a)+4 f\left(\frac{a+b}{2}\right)+f(b)\right] \\
& \quad-\frac{2^{\alpha-1} \Gamma(\alpha+1)}{(b-a)^{\alpha}}\left[I_{a^{+}}^{\alpha} f\left(\frac{a+b}{2}\right)+I_{b^{-}}^{\alpha} f\left(\frac{a+b}{2}\right)\right] \mid \\
& \quad \leq \frac{b-a}{2}\left(\int_{0}^{1}\left|\frac{t^{\alpha}}{2}-\frac{1}{3}\right| d t\right)^{1-1 / q} \\
& . \mid\left(\int_{0}^{1}\left|\frac{t^{\alpha}}{2}-\frac{1}{3}\right|\left|f^{\prime}\left(\frac{1+t}{2} b+\frac{1-t}{2} a\right)\right|^{q} d t\right)^{1 / q} \\
& +\left(\int_{0}^{1}\left|\frac{1}{3}-\frac{t^{\alpha}}{2}\right| d t\right. \\
& \left.\left..\left|f^{\prime}\left(\frac{1+t}{2} a+\frac{1-t}{2} b\right)\right|^{q} d t\right)^{1 / q}\right] .
\end{aligned}
$$


By the $h$-convexity of $\left|f^{\prime}\right|^{q}$ and using the fact that $\mid t^{\alpha} / 2-$ $1 / 3|=| 1 / 3-t^{\alpha} / 2 \mid \leq 1 / 3$ for all $t \in[0,1]$, we have

$$
\begin{aligned}
& \int_{0}^{1}\left|\frac{t^{\alpha}}{2}-\frac{1}{3}\right|\left|f^{\prime}\left(\frac{1+t}{2} b+\frac{1-t}{2} a\right)\right|^{q} d t \\
& \leq \frac{1}{3}\left(\left|f^{\prime}(b)\right|^{q} \int_{0}^{1} h\left(\frac{1+t}{2}\right) d t\right. \\
& \left.+\left|f^{\prime}(a)\right|^{q} \int_{0}^{1} h\left(\frac{1-t}{2}\right) d t\right), \\
& \int_{0}^{1}\left|\frac{1}{3}-\frac{t^{\alpha}}{2}\right|\left|f^{\prime}\left(\frac{1+t}{2} a+\frac{1-t}{2} b\right)\right|^{q} d t \\
& \leq \frac{1}{3}\left(\left|f^{\prime}(a)\right|^{q} \int_{0}^{1} h\left(\frac{1+t}{2}\right) d t\right. \\
& \left.+\left|f^{\prime}(b)\right|^{q} \int_{0}^{1} h\left(\frac{1-t}{2}\right) d t\right) .
\end{aligned}
$$

Using the last two inequalities in (21) we obtain (20). This completes the proof.

\section{Conflict of Interests}

The author declares that there is no conflict of interests regarding the publication of this paper.

\section{References}

[1] S. S. Dragomir, R. P. Agarwal, and P. Cerone, "On Simpson's inequality and applications," Journal of Inequalities and Applications, vol. 5, pp. 533-579, 2000.

[2] M. Z. Sarikaya, E. Set, and E. Özdemir, "On new inequalities of Simpson's type for convex functions," RGMIA Research Report Collection, vol. 13, no. 2, article 2, 2010.

[3] M. Z. Sarikaya, E. Set, and M. E. Özdemir, "On new inequalities of Simpson's type for s-convex functions," Computers \& Mathematics with Applications, vol. 60, no. 8, pp. 2191-2199, 2010.

[4] M. Alomari, M. Darus, and S. S. Dragomir, "New inequalities of Simpson's type for s-convex functions with applications," RGMIA Research Report Collection, vol. 12, no. 4, article 9, 2009.

[5] M. Alomari and S. Hussain, "Two inequalities of Simpson type for quasi-convex functions and applications," Applied Mathematics E-Notes, vol. 11, pp. 110-117, 2011.

[6] Z. Liu, "An inequality of Simpson type," Proceedings of The Royal Society of London. Series A. Mathematical, Physical and Engineering Sciences, vol. 461, no. 2059, pp. 2155-2158, 2005.

[7] E. Set, M. E. Özdemir, and M. Z. Sarikaya, "On new inequalities of Simpson's type for quasi-convex functions with applications," Tamkang Journal of Mathematics, vol. 43, no. 3, pp. 357-364, 2012.

[8] S. Varošanec, "On $h$-convexity," Journal of Mathematical Analysis and Applications, vol. 326, no. 1, pp. 303-311, 2007. 


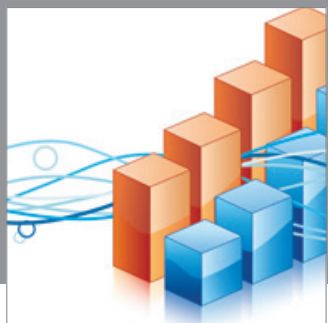

Advances in

Operations Research

mansans

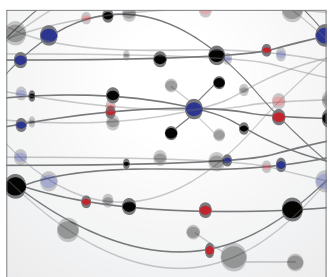

The Scientific World Journal
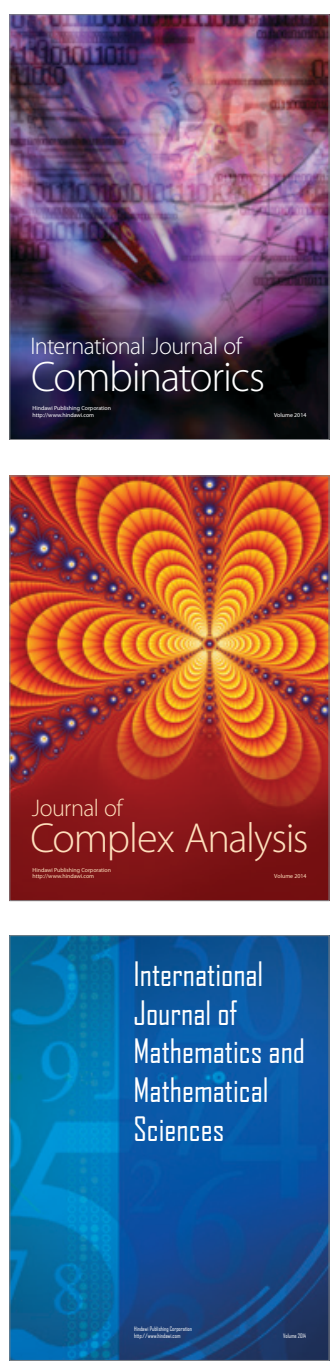
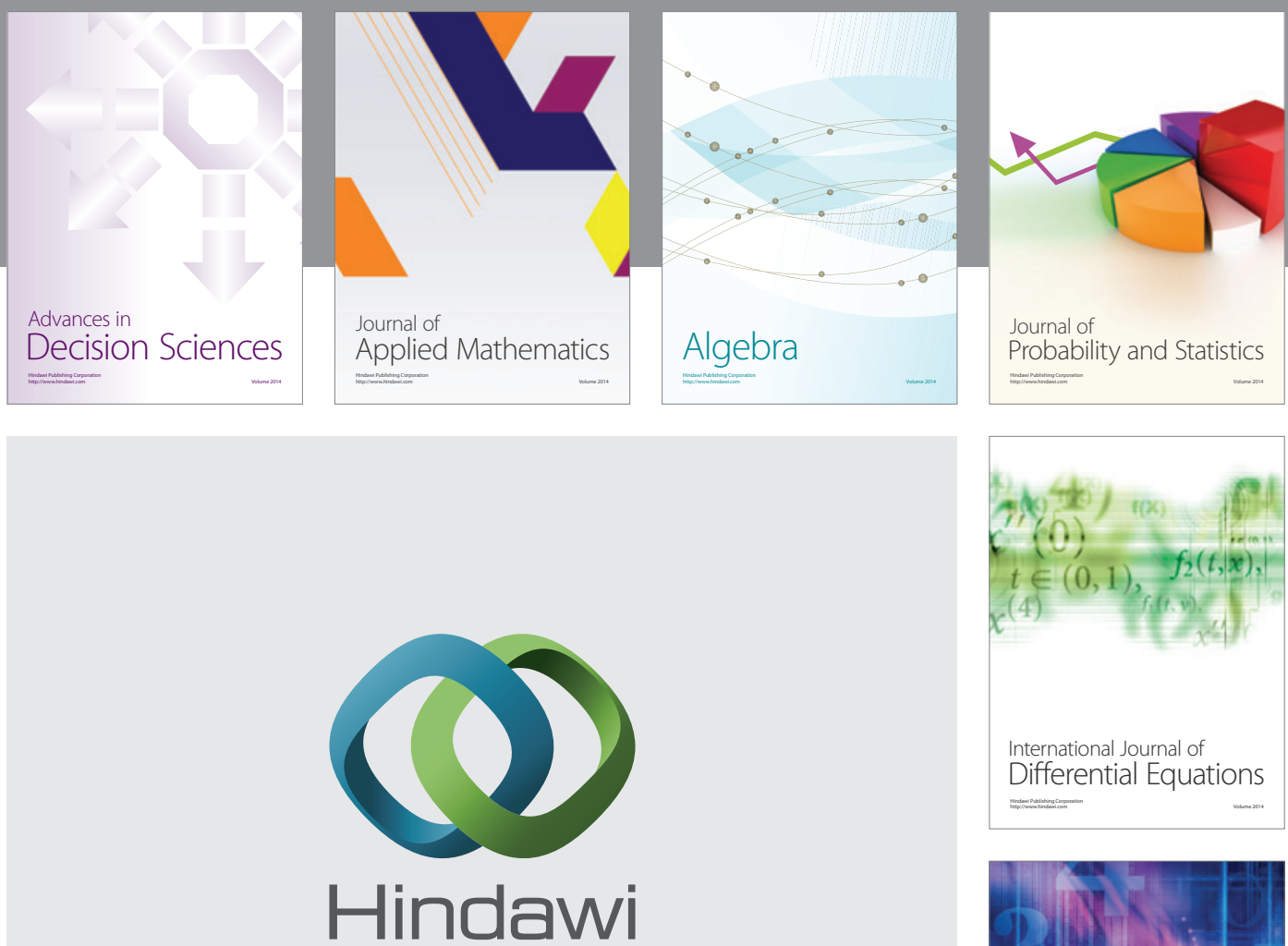

Submit your manuscripts at http://www.hindawi.com
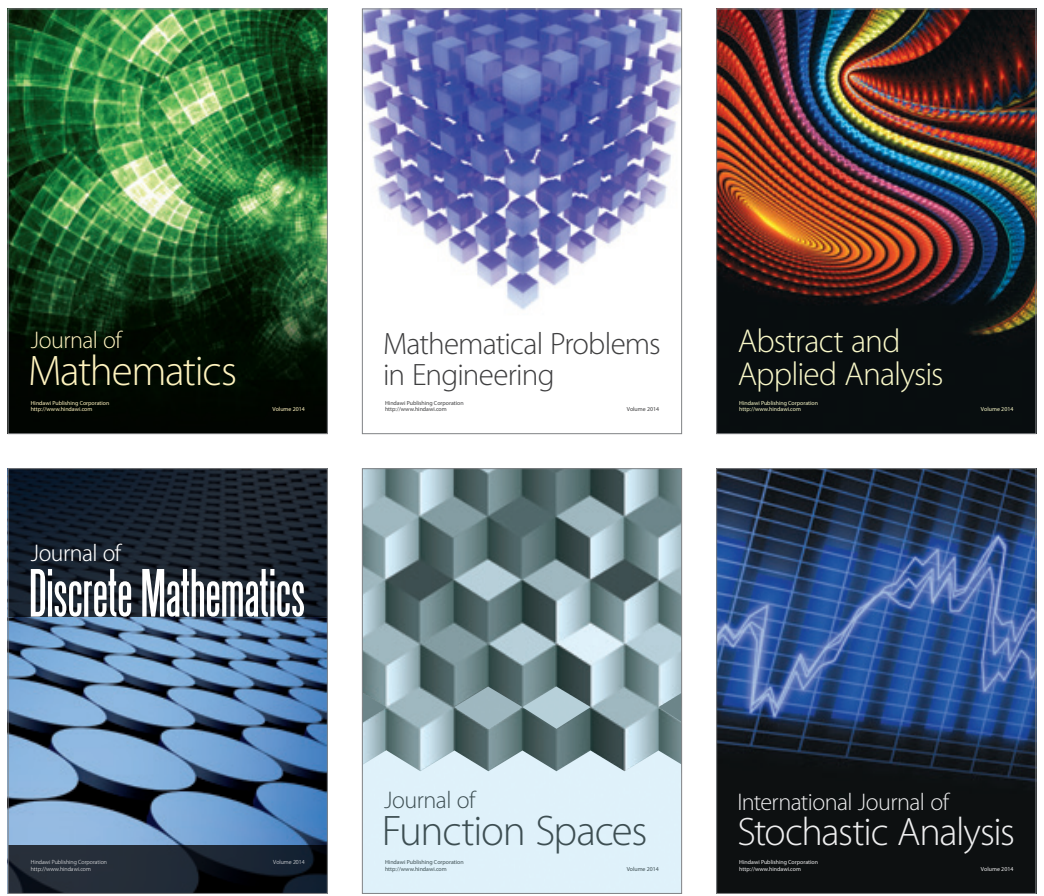

Journal of

Function Spaces

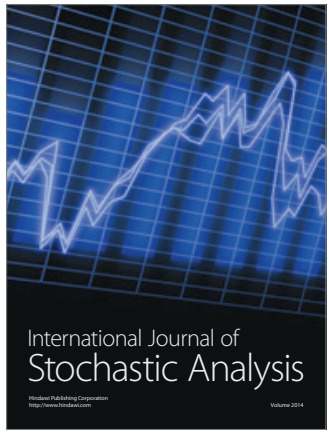

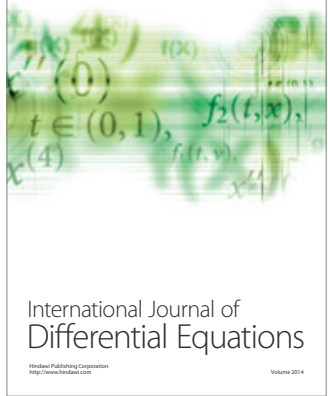
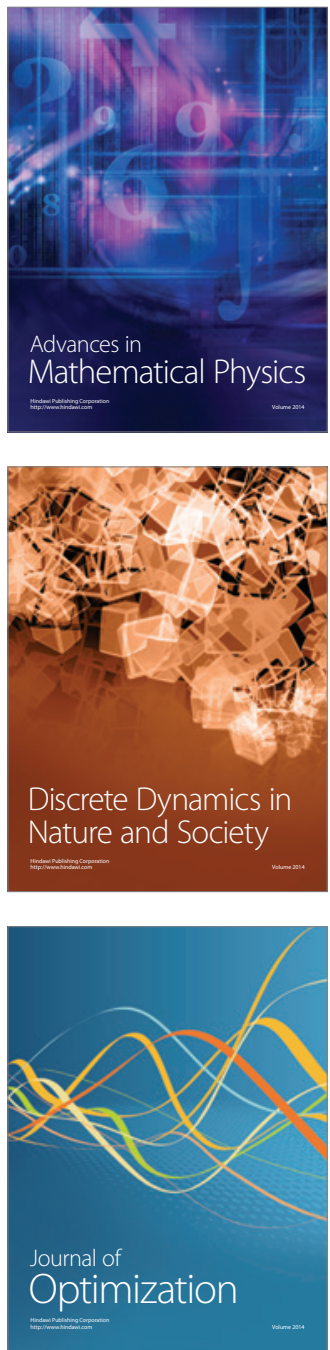